\title{
2nd generation DNA sequencing meets functional genomics
}

\section{Ivo G. Gut}

\section{Centro Nacional de Analisis Genomico, C/Baldiri Reixac 4, 08028 Barcelona, Spain}

Commentary on: Bernd Timmermann et al. A new dominant peroxiredoxin allele identified by whole-genome resequencing of random mutagenized yeast causes oxidant-resistance and premature aging. Aging 2010; 2: 475-486.

E-mail: igut@pcb.ub.es

The introduction of the first 2nd generation DNA sequencing method five years ago heralded a paradigm shift and propelled us into a new era [1]. In contrast to the Sanger sequencing method that dominated DNA sequencing for nearly 30 years [2], 2nd generation sequencing analyzes clonal representations of the input DNA. Using highly parallel processing in microfluidic devices and stepwise addition of four nucleobases highly accurate sequencing results are obtained. Orders of magnitude larger amounts of data are generated at substantially decreased cost and effort. This has made it possible to sequence a human genome completely in less than one month using one 2nd generation sequencing instrument. At first 2nd generation sequencers were used for experiments with reduced representation of the genome such as ChIPseq and RNAseq [3, 4]. These applications have now reached a very high level of sophistication and are replacing array-based versions of these kinds of analyses. The application of whole genome sequencing for genetic application has been slow on the uptake mainly due to cost, effort and resolution required. There are a few large international efforts to collect high value information such as for the 1000 Genomes Project and the International Cancer Genome Consortium (ICGC).

Timmermann et al. use complete sequencing of a mutagenized S. cervisiae strain with a longevity phenotype of interest to identify the functionally effected gene [5]. By a clever sequence pruning procedure they rapidly arrive at a manageable number of putative variants responsible for the alteration of the phenotype. The mutated variants of the candidate genes are introduced into wildtype $\mathrm{S}$. cerevisiae for identification and validation. Interestingly the causative variant of TSA1 does not result in a loss or gain but shows a modulation of function.
The approach developed here highlights a way for functional genomics making use of the extensive collections of mutants of different model organisms.

This functional analysis strategy could also be applied to reverse genetic characterization.

\section{REFERENCES}

1. Margulies M et al. Nature. 2005; 437:376-380.

2. Sanger F et al. Proc Natl Acad Sci U S A. 1977; 74:5463-5467.

3. Schmidt D et al. Methods. 2009; 48:240-248.

4. Wilhelm BT and Landry JR. Methods. 2009; 48:249-257.

5. Timmermann B et al. Aging. 2010; 2:475-486. 